\title{
Design Analysis and Applications of a 3D Laser Ball Bar for Accuracy Calibration of Multiaxis Machines
}

\author{
Kuang-Chao Fan, Dept. of Mechanical Engineering, National Taiwan University, Taipei, Taiwan, ROC \\ Hai Wang, Dept. of Mechanical Engineering, Ming-Chi Institute of Technology, Taipei, Taiwan, ROC \\ Fang-Jung Shiou, Dept. of Mechanical Engineering, National Taiwan University of Science and Technology, \\ Taipei, Taiwan, ROC \\ Chih-Wei Ke, Richip Co., Hsinchu, Taiwan, ROC
}

\begin{abstract}
Conventional techniques for measuring the volumetric errors of Cartesian coordinate machine tools are time consuming using a laser interferometer or step gauges. For multiaxis machines, where the spindle can swing, volumetric error calibration is even more difficult. In this study, a novel 3D laser ball bar (3D-LBB) has been developed for the easy setup and rapid measurement of the tool position relative to the worktable at any working point of multiaxis machines. The instrument makes use of one laser ball bar and two rotary laser encoders to detect the target path in the spherical coordinate system. The design of the instrument is discussed, and the error attributes are analyzed to enhance the instrument's accuracy. Applications to the volumetric error measurement of a robot and two types of machine tools have demonstrated the high-precision capability of this 3D laser ball bar.
\end{abstract}

Keywords: 3D Laser Ball Bar, Accuracy Calibration, Volumetric Errors, Multiaxis Machines

\section{Introduction}

Techniques for performing accuracy testing of CNC machine tools can be found in many standards, such as ISO 230 or ASME B5.54 (1993). Most of the existing linear measurement instruments are one dimensional, such as a laser interferometer or step gauge. For the circular test of 2-D motion, as specified in ISO 230-4 (1998), some instruments have been developed, such as the double ball bar (DBB) (Bryan 1982), Contisure (Burdekin and Jwye 1992), and the latest laser ball bar (LBB) (Ziegert and Mize 1994, Schmitz and Ziegert 2000). Although these instruments are capable of two-axis error measurements, they are still sensitive to one dimension only.

This paper is an expanded version of a paper originally presented at NAMRC 31 and published in the Transactions of NAMRI/SME, Vol. 31, 2003.
For the measurement of volumetric errors of machine tools, most methods detect 21 component errors and then use the homogeneous transformation matrix (HTM) method or kinematic analysis method (Soons, Theuws, and Schellenkens 1992) to derive the spatial errors off-line. Wang (2000) follows the ASME B5.54 standard to measure volumetric errors directly. The standard employs a laser Doppler displacement meter (LDDM) and a large, flat mirror to measure four body diagonals, and assesses volumetric errors using the vector method. The standard is time saving but only valid for traditional, serial-type CNC machines. Hexapod machine tools have received a great deal of attention due to their flexibility in five-axis movement (Patel and Ehmann 1997). However, owing to the spindle swing in the pitch and yaw directions, more sensors are needed to make the volumetric motion measurement possible (Parenti and Gregorio 1999). In practice, however, this equipment is too expensive to be implemented in industry. So far, there are some good laser tracking systems (LTSs) designed in spherical coordinate systems that can directly detect 3D motion error (API 2002). These systems must be operated in an active way, which requires feedback sensors and servo control to track the moving target in real time. These systems require very fast control systems, resulting in higher cost.

A novel design that integrates the merits of a LBB and LTSs is proposed in this research for the threedimensional measurement of moving objects in real time. The system, called the 3D Laser Ball Bar (3DLBB), is based on the spherical coordinate principle containing only one precision laser linear measurement device and two precision laser rotary encoders 


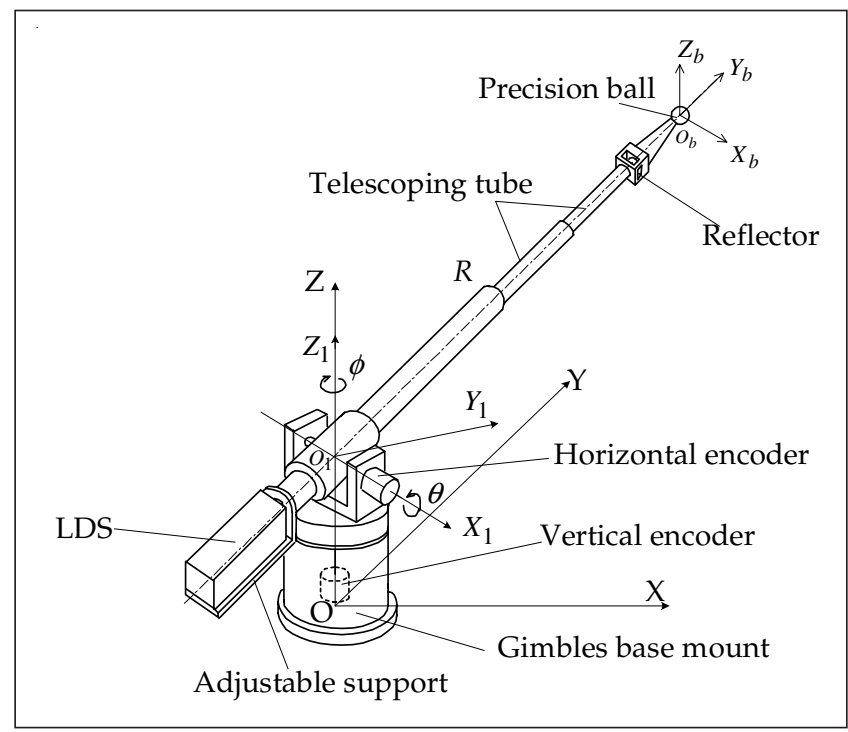

Figure 1

Structure of 3D Laser Ball Bar

in the gimbal base with an extendable ball bar. Such a system can be dragged by any 3D moving target with a magnetic head and freely moved in the space. Three sensors simultaneously record the ball positions and transform into the Cartesian coordinate in real time. Having been calibrated by a HP laser interferometer, the system accuracy can be compensated and enhanced to a higher degree. As this system is operated in the passive mode in $3 \mathrm{D}$ space, the cost is inexpensive.

\section{Design Principle of 3D Laser Ball Bar (3D-LBB)}

\section{Structure Design}

Figure 1 illustrates the system configuration of the 3D-LBB. The system is constructed in a spherical coordinate of which the center of the gimbal mount is the origin $(O)$. Mounted onto the gimbal center is an extendable telescoping tube (made of aluminum alloy and hardened in the interior surface) that can be designed in two or three sections depending on the required radial length of motion. Between the inner and outer tubes of a sliding pair is one linear bearing mounted at the far end of the outer tube and a copper sliding bearing fixed at the near end of the inner tube to prevent side motion during bar extension.

The movement of the 3D-LBB is generated by the precision end ball, which can be dragged by a magnetic socket carried by any moving object. The radial motion $(R)$ of the ball is detected by a small-

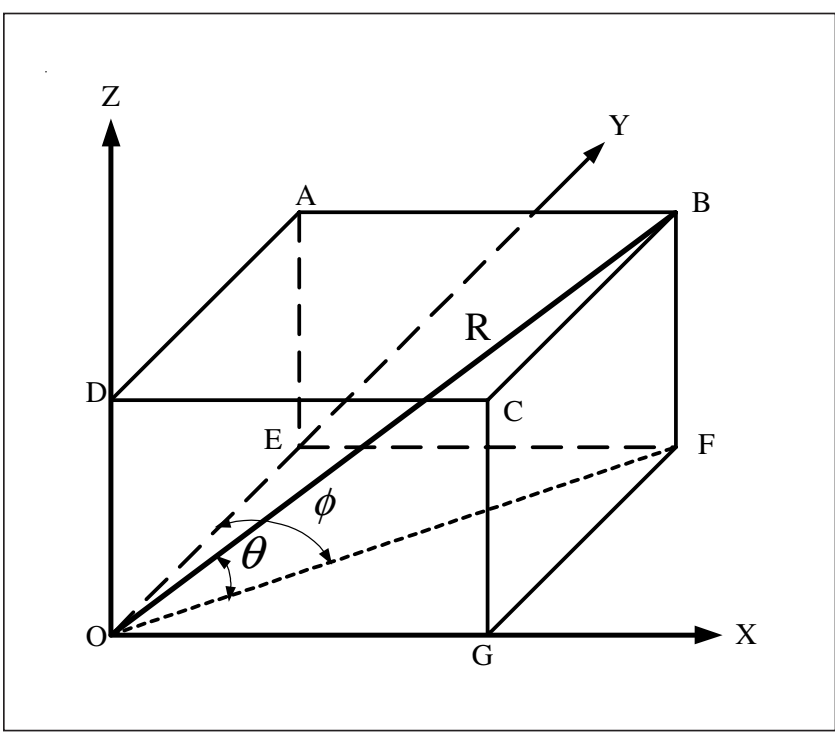

Figure 2

Spherical and Cartesian Coordinates

sized laser linear measurement system whose beam passes through the telescope tubes and is reflected back by a reflector at the bar end. A conical stainless steel piece connecting the reflector and the ball allows a wider angle of rotation between the magnetic socket and the ball. The laser linear system is required to be as small as possible to reduce the system weight. This design was, therefore, changed to a compact laser Doppler scale (LDS, model 109N, made by Optodyne Co.) with wavelength stability to $0.1 \mathrm{ppm}$ and system accuracy to $1.0 \mathrm{ppm}$.

The pitch $(\theta)$ and the yaw $(\phi)$ motions of the bar with respect to the gimbal base are detected by two precision laser rotary encoders (model K-1, made by Canon Co.) individually. Each encoder has very fine scales of 81,000 ppr. With an additional 16-division interpolator board (model 16-2), the resolution can reach one arc-sec.

\section{Coordinate Transformation}

To obtain the tool-point Cartesian coordinates, the equations of coordinate transformation can be easily derived as shown in Figure 2.

$$
\left.\begin{array}{c}
X=R \cos \theta \sin \phi \\
Y=R \cos \theta \cos \phi \\
Z=R \sin \theta
\end{array}\right\}
$$

where $R=R_{0}+r . R_{0}$ is the initial length of the bar, which is obtained during the initialization process, as explained in a later section, and $r$ is the extended 


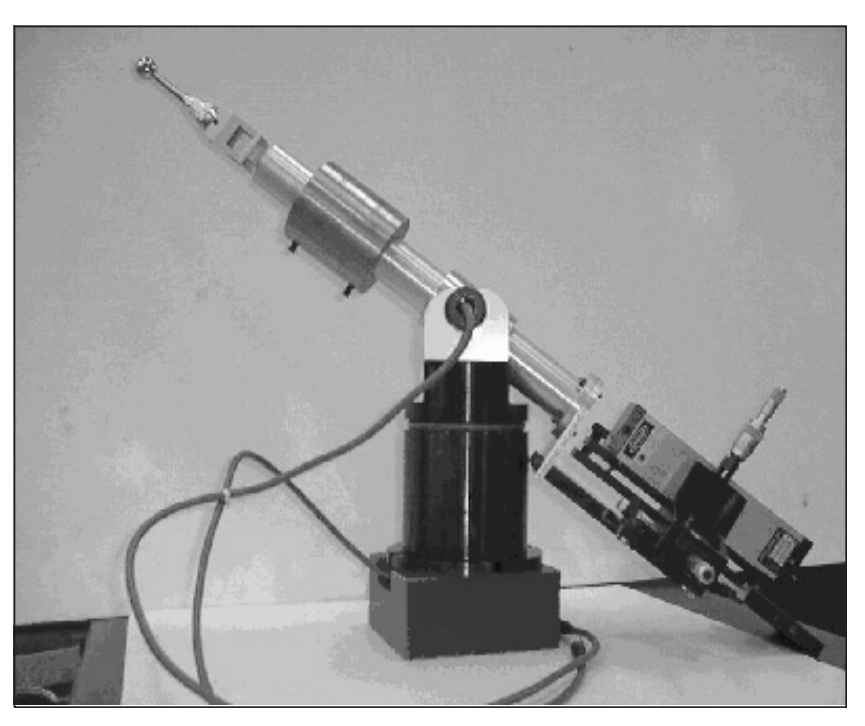

Figure 3

Prototype of 3D-LBB

length. Figure 3 shows the picture of the developed prototype 3D-LBB. The nominal physical dimensions of this prototype are: gimbal base diameter 150 $\mathrm{mm}$, gimbal center height $300 \mathrm{~mm}$, initial $R$ length $142 \mathrm{~mm}$, and moving length of telescope tubes 550 $\mathrm{mm}$. To balance the weight while the telescope tube is extending, a counterweight balancer is mounted onto the base tube near the origin. Moreover, for ease of beam alignment, a four-axis adjustable stage is especially made for laser mounting.

\section{Accuracy Considerations}

As an instrument for spatial position measurement, the system must be more accurate than the inspected multiaxis machine itself. Error sources, therefore, must be identified and properly calibrated to improve the instrument's system accuracy.

\section{Clearance Error of Tubes}

As shown in Figure $4 a$, under improper fit of the linear bearing a small amount of clearance may generate side motion during the extension of the inner tube.

The angle of rotation can be estimated by $\tan \theta=$ $2 C / L$, where $C$ denotes clearance and $L$ indicates bearing length. In this study, a copper spacer was designed to give interference fit with the end of the inner tube and precise fit with the outer tube. Under good lubrication, the copper spacer can freely slide along the outer tube as a slide bearing. Because in

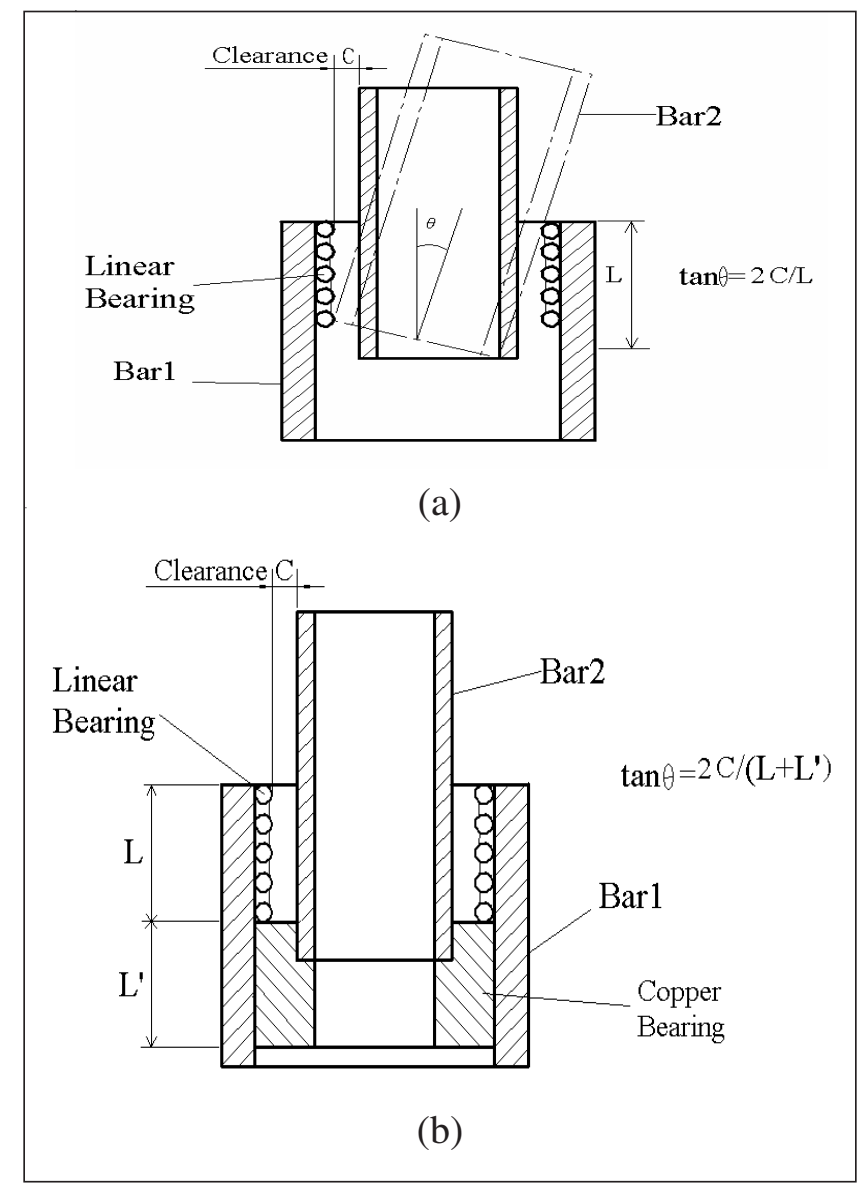

Figure 4

Clearance Error: (a) caused by linear bearing, (b) corrected by a copper bearing.

practice the linear bearing and tubes have to be selected from standard items in the shop, a possible clearance even after minor machining still cannot be avoided. The copper spacer, however, can be selfmade to assure minimum clearance. Therefore, even at the maximum extension condition, as shown in Figure $4 b$, the loose motion of the inner tube must be much smaller than before.

\section{Assembly Errors}

To modify the measurement inaccuracy, geometric assembly errors should be superimposed to Eq. (1). These include the axes perpendicularity and the axes offset with respect to the origin $(O)$ of the reference frame $X Y Z$. These errors are illustrated in Figures 5-7, respectively.

As shown in Figure 5, the perpendicularity error $(\alpha)$ of the $\theta$-axis with respect to the $Z$ axis can be checked by a CMM and computed as 


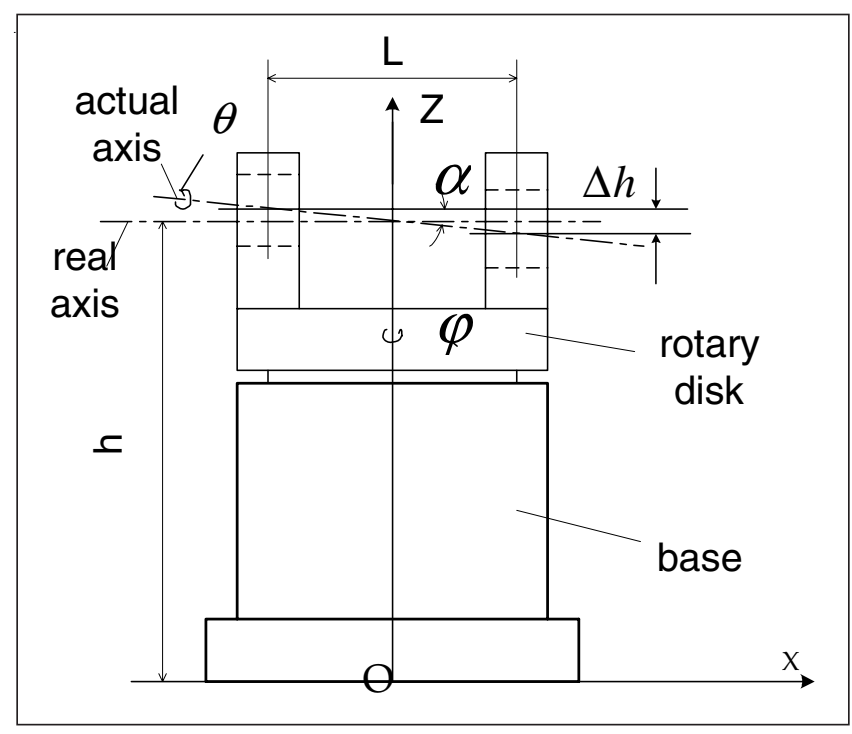

Figure 5

Squareness Error of $\theta$-Axis to $Z$-Axis

$$
\alpha=\arctan \frac{\Delta h}{L}
$$

where $\Delta h$ is the difference of the height of two bearing centers and $L$ is the distance between two bearing supports. The measurement can be carried out before mounting the shaft and the encoders. The measured inner ring surface should be as close to the outside wall as possible. The length $L$ is determined by the distance between the two outside walls of the bearing supports. Another way is to measure the wobble of the rotary disk. Placing a dial gauge at $100 \mathrm{~mm}$ radial position and rotating the disk a full circle, the height change of the disk surface could be found. Using Eq. (2), the perpendicularity error $(\alpha)$ is obtained.

From Figure 6, the perpendicularity error $(\beta)$ of the bar with respect to the $\theta$-axis also can be checked by the CMM. The origin $O_{1}$ indicates the intersection of the $\theta$-axis and $R$-axis. From Figure $7 a$, in the $X-Y$ plane there is an offset error $(a, b)$ of origin $O_{1}$ with respect to origin $O$ of the $\phi$-axis. The $(a, b)$ offset could be found by the following procedures:

1. Use a roundness measuring machine to measure the central circle of the rotary disk and find the offset $\left(a_{1}, b_{1}\right)$ of center $O_{o}$ with respect to the rotation center $O$ (refer to Figure 5).

2. Use the CMM to find the offset $\left(a_{2}, b_{2}\right)$ of the disk center $O_{o}$ with respect to the origin $O_{1}$.

3. Therefore, $a=a_{2}-a_{1}, b=b_{2}-b_{1}$.

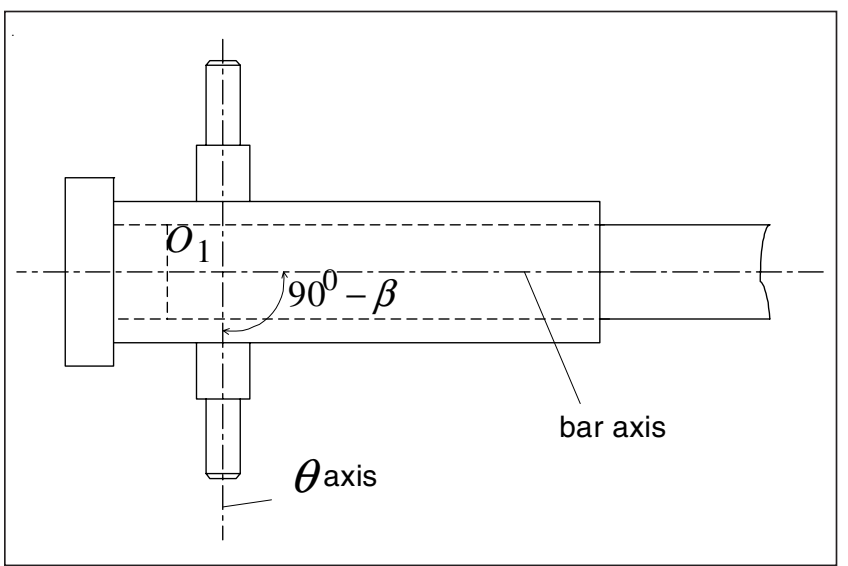

Figure 6

Squareness Error $\theta$-Axis

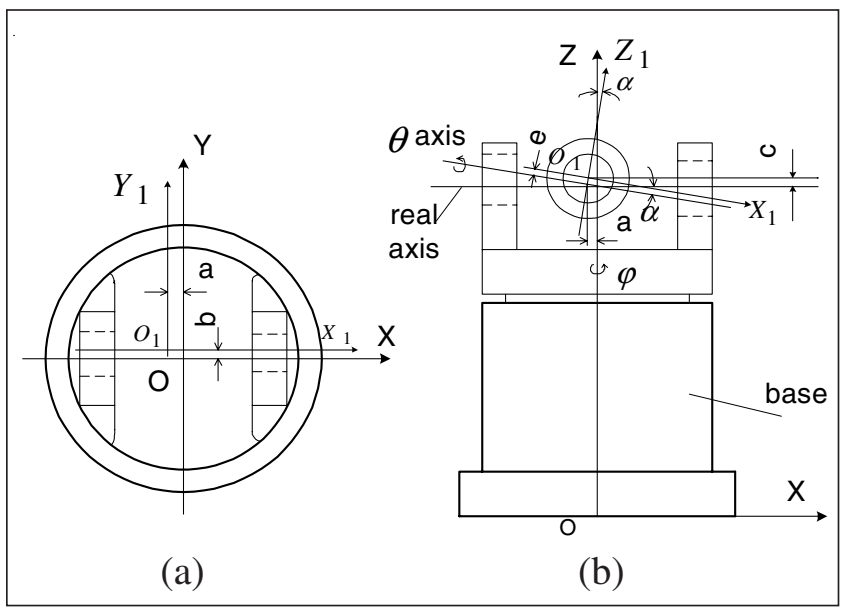

Figure 7

Eccentricity of Origin $O_{1}$

Then, in the Z-direction, the deviation $c$ (see Figure $7 b$ ) can be calculated as

$$
c=a \tan \alpha+e \cos \alpha
$$

where $e$ is the center offset of the bar axis with respect to the $\theta$-axis along the $Z_{1}$ direction.

Experimental tests were conducted on the prototype 3D-LBB. All the assembly errors could thus be identified as listed in Table 1.

Because $a, b, c, \alpha$, and $\beta$ are constant assembly errors, these factors must be added to the system mechanism, and Eq. (1) has to be modified accordingly. To derive the actual measurement equations, two relative coordinate frames are of interest, as shown in Figure 8. The reference frame $X Y Z$ is fixed at the base center, with the $Z$-axis denoting the cylindrical base axis. A movable frame $X_{1} Y_{1} Z_{1}$ is assigned at the point $O_{1}$, with $X_{1}$ parallel to the $\theta$-axis and $Y_{1}$ 
Table 1

Prototype 3D-LBB Assembly Errors

\begin{tabular}{ccccc}
\hline $\mathrm{a}$ & $\mathrm{b}$ & $\mathrm{c}$ & $\alpha$ & $\beta$ \\
$0.024 \mathrm{~mm}$ & $0.018 \mathrm{~mm}$ & $0.008 \mathrm{~mm}$ & 45 arc-sec. & 86 arc-sec. \\
\hline
\end{tabular}

parallel to the $X-Y$ plane. Equation (1) can be modified in terms of the homogeneous transformation matrix (HTM) as follows:

$$
\begin{gathered}
{ }^{\circ} O_{b}={ }^{o}[T]_{b}=[\phi] \cdot[\Delta] \cdot[\alpha] \cdot[\beta] \cdot[\theta] \cdot[R] \\
{[\phi]=\left[\begin{array}{cccc}
\cos \phi & \sin \phi & 0 & 0 \\
-\sin \phi & \cos \phi & 0 & 0 \\
0 & 0 & 1 & 0 \\
0 & 0 & 0 & 1
\end{array}\right] ;[\Delta]=\left[\begin{array}{cccc}
1 & 0 & 0 & a \\
0 & 1 & 0 & b \\
0 & 0 & 1 & c+h \\
0 & 0 & 0 & 1
\end{array}\right] ;} \\
{[\alpha]=\left[\begin{array}{cccc}
\cos \alpha & 0 & \sin \alpha & 0 \\
0 & 1 & 0 & 0 \\
-\sin \alpha & 0 & \cos \alpha & 0 \\
0 & 0 & 0 & 1
\end{array}\right] ;[\beta]=\left[\begin{array}{cccc}
\cos \beta & \sin \beta & 0 & 0 \\
-\sin \beta & \cos \beta & 0 & 0 \\
0 & 0 & 1 & 0 \\
0 & 0 & 0 & 1
\end{array}\right]} \\
{[\theta]=\left[\begin{array}{cccc}
1 & 0 & 0 & 0 \\
0 & \cos \theta & -\sin \theta & 0 \\
0 & \sin \theta & \cos \theta & 0 \\
0 & 0 & 0 & 1
\end{array}\right] ;[R]=\left[\begin{array}{cccc}
1 & 0 & 0 & 0 \\
0 & 1 & 0 & R \\
0 & 0 & 1 & 0 \\
0 & 0 & 0 & 1
\end{array}\right]}
\end{gathered}
$$

where ${ }^{\circ} O_{b}$ denotes the spatial position of the ball center with respect to reference frame $X Y Z$ and $h$ is the actual height of origin $O_{1}$ of frame $X_{1} Y_{1} Z_{1}$. Therefore, the actual ball position in space during motion can be calculated with the following equation, Eq. (5).

$$
\begin{aligned}
& X=\left(\begin{array}{l}
(\cos \phi \cos \alpha \sin \beta+\sin \phi \cos \beta) \cos \theta \\
+\cos \phi \sin \alpha \sin \theta
\end{array}\right) R \\
& +a \cos \phi+b \sin \phi \\
& Y=\left(\begin{array}{l}
(-\sin \phi \cos \alpha \sin \beta+\cos \phi \cos \beta) \cos \theta \\
-\sin \phi \sin \alpha \sin \theta
\end{array}\right) R \\
& -a \sin \phi+b \cos \phi \\
& Z=(-\sin \alpha \sin \beta \cos \theta+\cos \alpha \sin \theta) R+c+h
\end{aligned}
$$

\section{Elastic Deformation}

Elastic deformation due to the moving bar center and dragging force at the ball is also the essential factor causing measurement inaccuracy. In this instrument, a balance sleeve was added to effectively

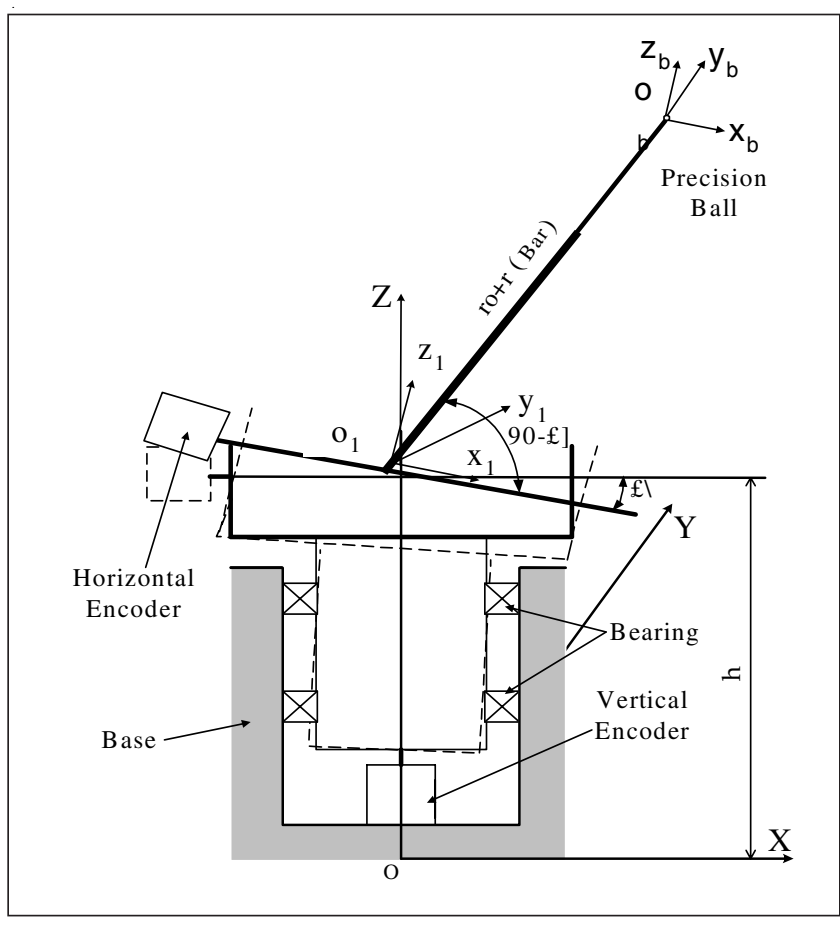

Figure 8

Structural Assembly Errors

reduce the deformation, as shown in Figure 3. The location and the weight of the balancer are determined such that, at no magnetic drag, when the bar is extended to half of the total length it reaches the equilibrium condition. Experiments showed that while the bar is extended to its full length or retracted to its shortest length, the small net force variation can be easily absorbed by the magnetic socket.

\section{Initialization of the 3D-LBB}

The initial length $\left(R_{0}\right)$ of the tube has to be identified with an initialization process. The initialization device consists of three sockets, as shown in Figure 9. $L_{1}$ and $L_{2}$ are the lengths of $\overline{A B}$ and $\overline{A C}$, respectively. Let point $\mathrm{A}$ be the reference point $\left(R=R_{0}, \phi=\right.$ $\left.\phi_{0}, \theta=\theta_{0}\right)$. The fixed distances of $L_{1}$ and $L_{2}$ are calibrated in advance by a high-precision CMM. The initialization procedure is as follows:

1. If the coordinates of each socket are measured, respectively, as $\mathrm{A}, \mathrm{B}\left(R_{0}+\Delta R_{\mathrm{B}}, \phi_{0}+\Delta \phi_{\mathrm{B}}, \theta_{0}+\right.$ $\left.\Delta \theta_{\mathrm{B}}\right)$, and $\mathrm{C}\left(R_{0}+\Delta R_{\mathrm{C}}, \phi_{0}+\Delta \phi_{\mathrm{C}}, \theta_{0}+\Delta \theta_{\mathrm{C}}\right)$, the following relations exist.

$$
\begin{aligned}
& |\overrightarrow{B O}-\overrightarrow{A O}|=L_{1} \\
& |\overrightarrow{C O}-\overrightarrow{A O}|=L_{2}
\end{aligned}
$$




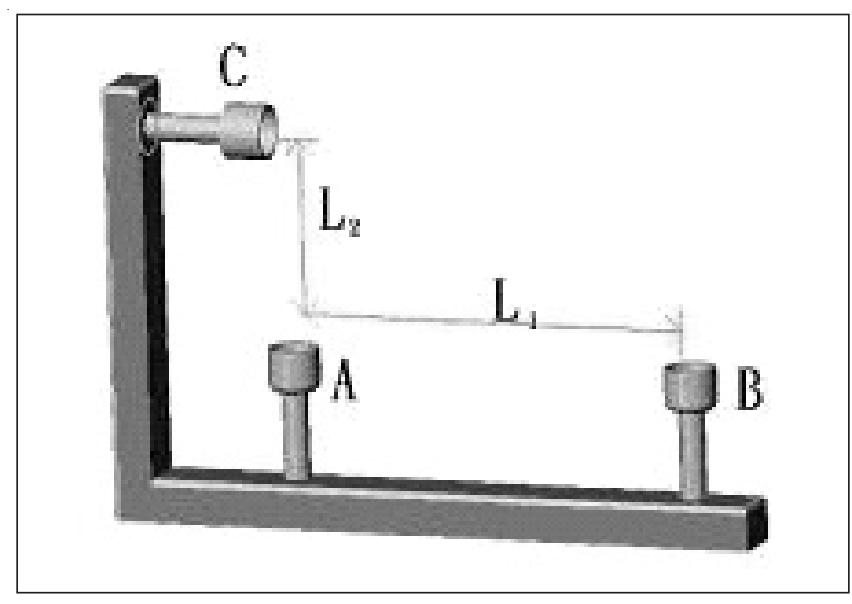

Figure 9

Artifact for 3D-LBB Initial Length Measurement

Substituting each coordinate into Eq. (6) and simplifying with trigonometric operations, the following two equations can be obtained.

$$
\begin{aligned}
L_{1}^{2}= & 2 R_{0}^{2}+\left(\Delta R_{B}\right)^{2}+2 R_{0} \Delta R_{B}- \\
& 2 R_{0}\left(R_{0}+\Delta R_{B}\right)\left(\cos \Delta \phi_{B}+1\right) \cos \Delta \theta_{B} \\
L_{2}^{2}= & 2 R_{0}^{2}+\left(\Delta R_{C}\right)^{2}+2 R_{0} \Delta R_{C}- \\
& 2 R_{0}\left(R_{0}+\Delta R_{C}\right)\left(\cos \Delta \phi_{C}+1\right) \cos \Delta \theta_{C}
\end{aligned}
$$

There is an interesting finding that in these two equations there is only one variable $R_{0}$. It means that only one standard distance is needed to find the initial bar length. The second standard distance can be used to double check the calculated $R_{0}$, which was found to be $142 \mathrm{~mm}$, as indicated earlier.

2. During the $R$-direction alignment step, first we let the precision ball of the 3D-LBB be dragged with a magnetic socket by the moving axis along the $R$ direction. Second, we adjusted the gimbal base to allow for two unchanged encoder readings throughout the travel. Then, we reset the two encoder readings.

3. Then we positioned the artifact in front of the 3D-LBB and let point $\mathrm{A}$ be in line with the moving axis as close as possible. Now we could take readings of $\mathrm{A}, \mathrm{B}$, and $\mathrm{C}$ sockets and found $R_{0}$.

In addition to the geometric errors by assembly and the elastic deformation as described in the above sections, there are also other systematic errors that affect the accuracy of measured $R, \phi$, and $\theta$ data,

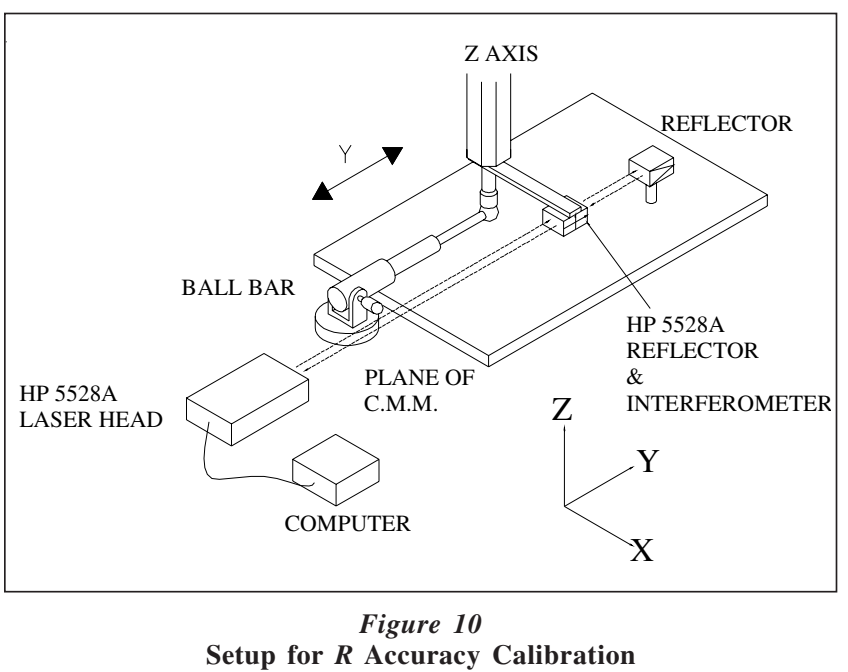

such as friction, laser stability and misalignment, clearances of fits, and sensor errors. Therefore, the accuracy calibration of the developed 3D-LBB is necessary. In addition, with the error compensation scheme the system accuracy can be significantly enhanced.

\section{Calibration of the $\boldsymbol{R}$ Axis}

As shown in Figure 10, an HP 5528A laser interferometer was used to compare with the reading of the LDS (not shown in the figure). The 3D-LBB was mounted onto a side table of a table-type CMM. The standard ball of the 3D-LBB was fixed to the spindle end with a magnetic socket. The set of corner cube reflectors and the interferometer cube were mounted on an extension bar from the spindle end. A possible Abbe error induced by this setup will be due to the roll error of the moving spindle. In fact, this roll error is very small and can be neglected. Bidirectional motion with three runs each was carried out for calibration. Calibrated results are shown in Figure $11 a$. Because the error has an obvious tendency and repeatability, the $R$ errors can be compensated by the best-fitting line. The slope of the error tendency is caused by the misalignment of the LDS laser beam with respect to the HP5528 laser beam. However, the maximum error at the far end is only about $1.5 \mu \mathrm{m}$, which can be reasonably compensated by a curve-fitting technique. The modified $R$ reading is expressed by Eq. (8). As shown in Figure $11 b$, after compensation, positioning errors of the LDS can be maintained within $\pm 0.3 \mu \mathrm{m}$.

$$
R_{\mathrm{mod}}=R_{r}-4 \times 10^{-6} R_{r}-0.0003
$$




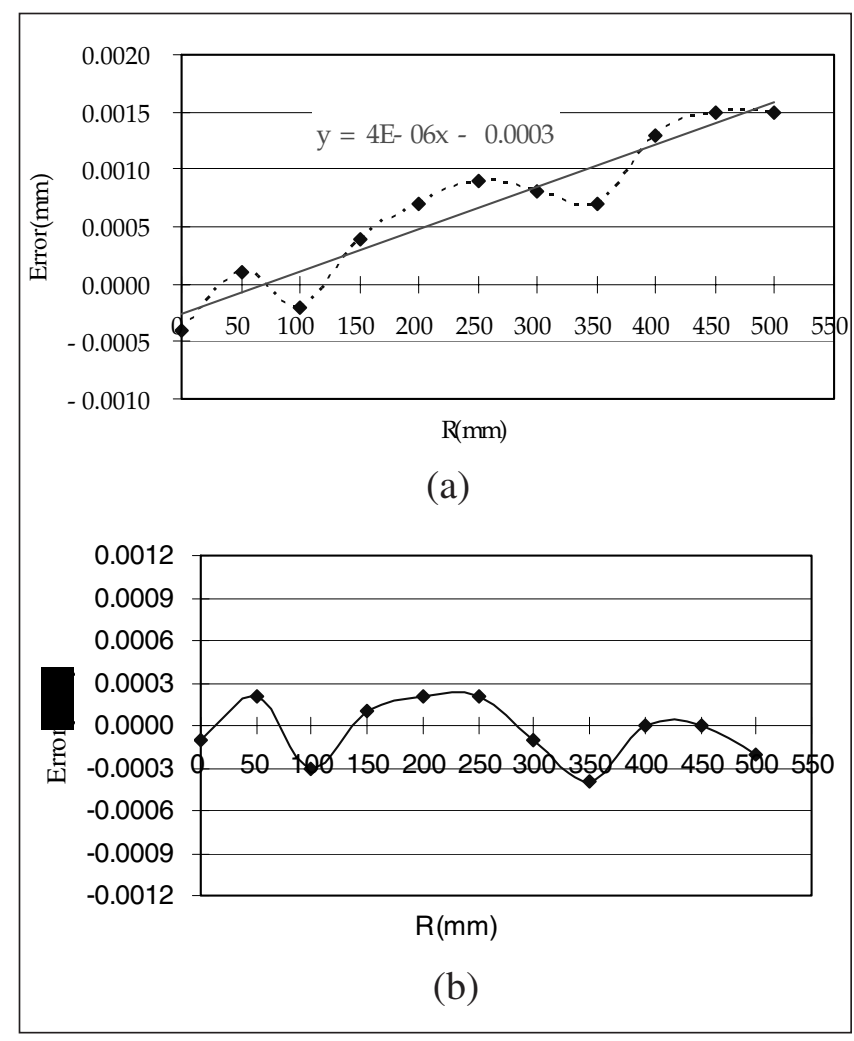

Figure 11

Calibrated $R$ Linear Errors

(a) results, (b) residual errors after compensation

where $R_{\text {mod }}$ is the modified $R_{r}$ value, which is the direct reading from the LDS.

\section{Calibration of the $\psi$ Axis}

In this experiment, the bar was carefully aligned parallel with the $X-Y$ plane and directed to the $Y$-axis of a table-type precision CMM, as shown in Figure $12 a$. The setup was similar to the setup of linear calibration, the only change being that the 3D-LBB moves to the $Y$-direction. While the spindle moves in the $X$-direction and its position is recorded by the HP 5528A, the $\phi$ angle changes in a trigonometric relationship, as shown in Figure $12 b$ and expressed by Eq. (9).

$$
\begin{aligned}
& \phi_{i}=\arccos \left(\frac{R_{0}^{2}+R_{i}^{2}-X_{i}^{2}}{2 R_{0} R_{i}}\right) \\
& \Delta \phi_{i}=\phi_{i r}-\phi_{i}
\end{aligned}
$$

where $\phi_{i}$ is the calculated nominal angle at the $i$ th position and $\phi_{i r}$ is the actual readout from the vertical encoder.

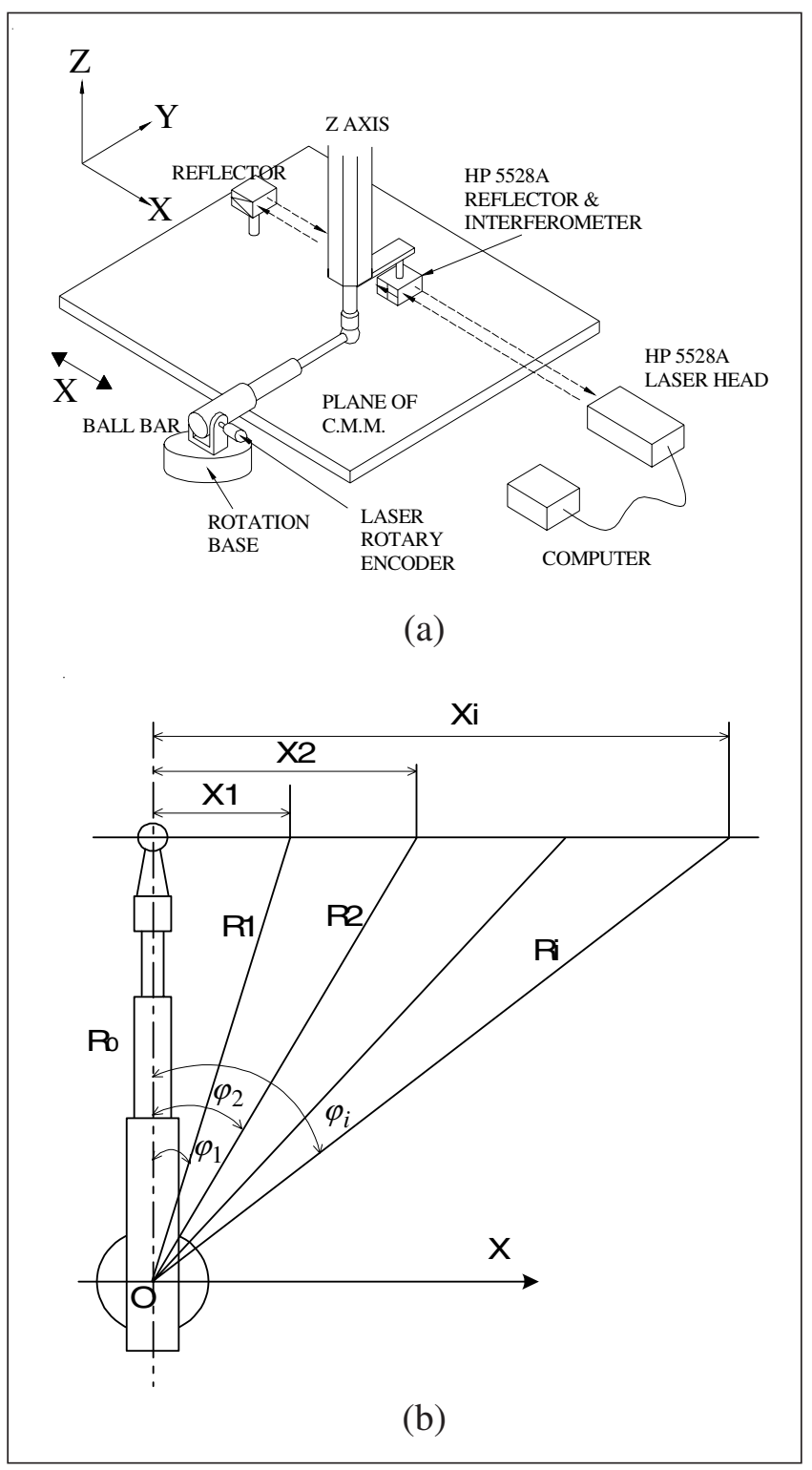

Figure 12

(a) Setup for $\phi$ calibration, (b) principle of calibration

The calibrated $\phi_{i}$ errors can be expressed by Eq. (10). After correction, the $\phi$ errors can be maintained within \pm 1.5 arc-sec, as shown in Figure 13 .

$$
\phi_{\text {mod }}=\phi_{r}-\left(-4 \times 10^{-5} \phi_{r}-23.818\right)
$$

where $\phi_{\text {mod }}$ is defined as the modified value and $\phi_{r}$ is the readout from the vertical encoder.

\section{Calibration of the $\theta$ Axis}

For calibration of the $\theta$ axis, the setup and principle of calibration are similar to Figure 12 except 


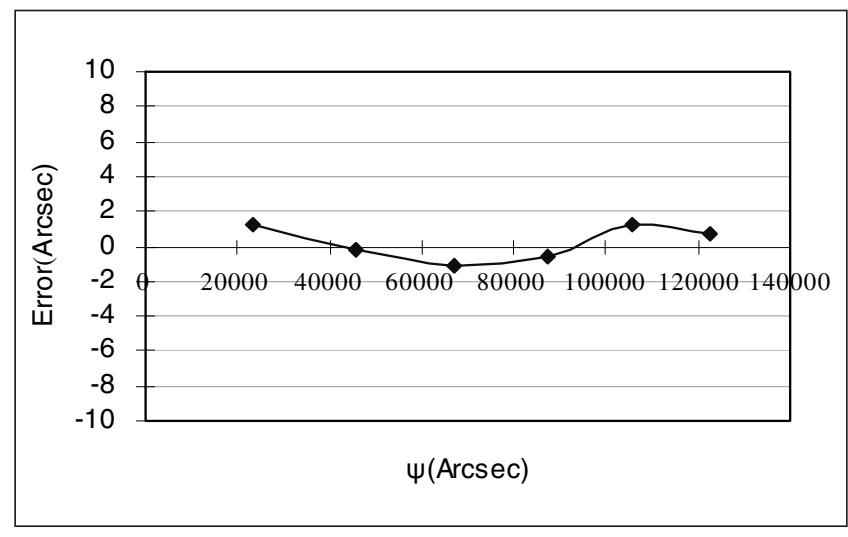

Figure 13

Calibrated $\phi$ Angular Errors

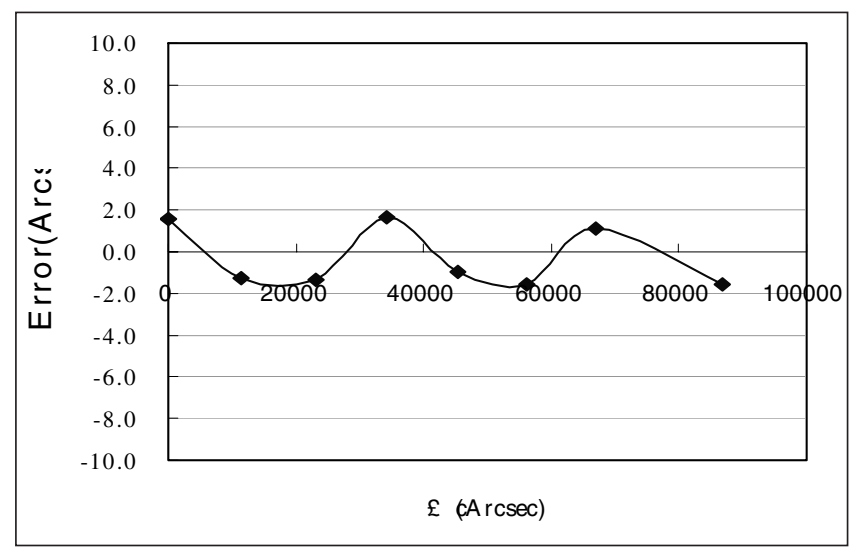

Figure 14

Calibrated $\theta$ Angular Errors

the laser beam is bent to the spindle direction. With proper alignment, the bar rotates only around the $\theta$ axis. The errors can be found by comparing the $\theta$ readouts from the horizontal encoder with values of $Z_{i}$ from HP and $R_{i}$ from LDS and calculated using Eq. (11). Error correction is done by Eq. (12). Residual errors are about \pm 1.6 arc-sec, as shown in Figure 14 .

$$
\begin{aligned}
& \theta_{i}=\arccos \left(\frac{R_{0}^{2}+R_{i}^{2}-Z_{i}^{2}}{2 R_{0} R_{i}}\right) \\
& \Delta \theta_{i}=\theta_{i r}-\theta_{i}
\end{aligned}
$$

where $\theta_{i}$ is the calculated nominal angle and $\theta_{i r}$ is the readout from the horizontal encoder.

$$
\theta_{\text {mod }}=\theta_{r}-\left(-0.0038 \times 10^{-5} \theta_{r}-3.1053\right)
$$

where $\theta_{\text {mod }}$ is the modified value and $\theta_{r}$ is the readout of the horizontal encoder.
Table 2

Accuracy and Repeatability of 3D-LBB

\begin{tabular}{cccc}
\hline Coordinate Parameter & Range & Accuracy & Repeatability $\pm 3 \sigma$ \\
\hline$R$ & $500 \mathrm{~mm}$ & $\pm 0.3 \mu \mathrm{m}$ & $0.21 \mu \mathrm{m}$ \\
$\phi$ & $35^{\circ}$ & $\pm 1.5 \mathrm{in}$. & $1.2 \mathrm{in.}$ \\
$\theta$ & $25^{\circ}$ & $\pm 1.6 \mathrm{in}$. & $0.8 \mathrm{in}$. \\
\hline
\end{tabular}

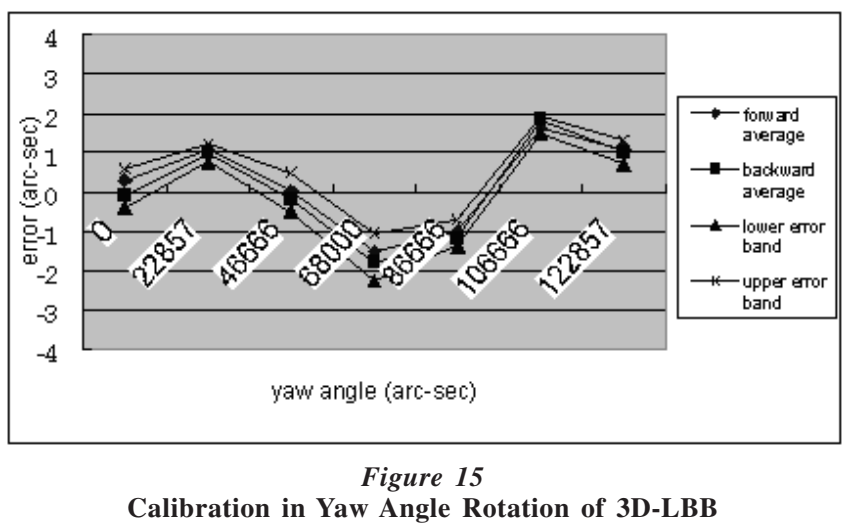

\section{Compensated 3D-LBB Errors}

After correction of the errors, the measurement accuracy of this instrument is significantly improved. The compensated system accuracy is listed in Table 2. Repeatability tests were based on the results of bidirectional motion with three runs each for all of the above three calibration processes. With careful assembly and fastening processes, the 3D-LBB can perform good repeatability. One typical example is shown in Figure 15 for the $\phi$ accuracy calibration. The uncertainties of all calibrated errors in the \pm 3 sigma band ( $99.7 \%$ certainty) are summarized in the last column of Table 2 .

\section{Applications}

\section{Volumetric Error Measurement of a Serial- Parallel Type Machine Tool}

The experimental setup is shown in Figure 16. The base of the 3D laser ball bar is fixed on the $X-Y$ table. The end ball is mounted at the spindle end by a magnetic socket with three points of contact. The machine tool is of a serial-parallel type consisting of a 3DOF parallel spindle platform with two angular orientations and one linear motion in the $Z$-axis and a conventional $X-Y$ table, which carries the workpiece. The spindle is assembled on the platform, which is connected to three constant-length struts by means of ball joints, which are equally spaced at 120 de- 


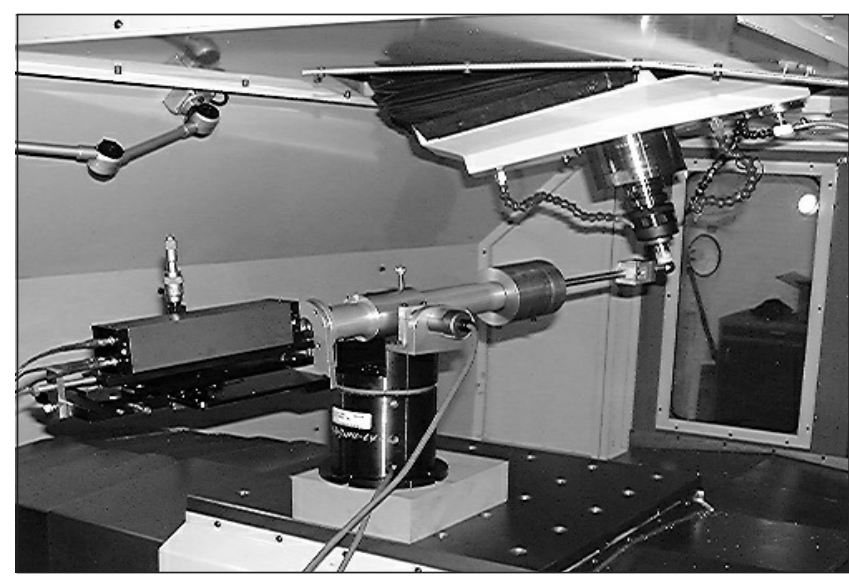

Figure 16

Setup for Accuracy Check of a Serial-Parallel Machine Tool

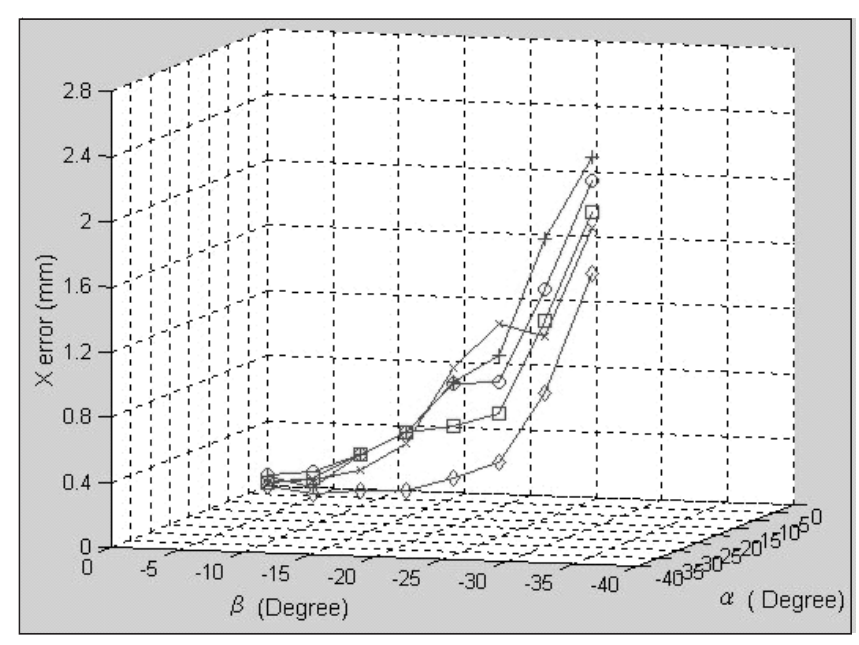

Figure 17

Diagonal Positioning Error Plot

grees. By giving commands to change two angular orientations of the spindle platform, the tool tip spatial positions could be measured by the 3D laser ball bar. Partial experimental results of volumetric errors are plotted in Figure 17.

\section{Accuracy Calibration and Verification in Diago- nal Path Measurement}

A diagonal path with 12 constant intervals of a machining center was measured by the $3 \mathrm{D}-\mathrm{LBB}$ and an HP interferometer conforming to the ASME B5.54 standard with machine coordinates from $(0,0,0)$ to $(312,324,128)$. Measured results are compared in Figure 18, from which it can be seen that this machine tool has significant volumetric errors, and the maximum difference between the 3D-LBB and HP5528 readouts is about $2.6 \mu \mathrm{m}$. This can verify the applicability of the developed 3D-LBB.

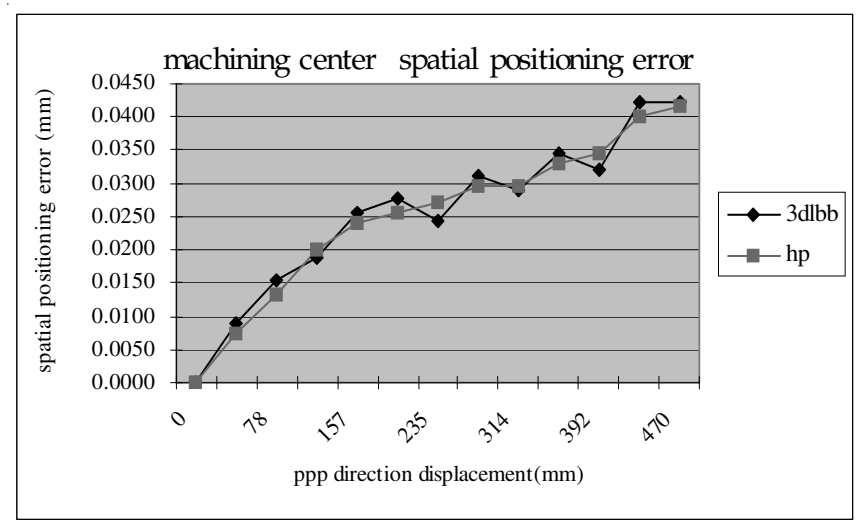

Figure 18

Comparison of Calibrated Results Between 3D-LBB and an HP 5528 Along a Diagonal Test of a Machine Tool

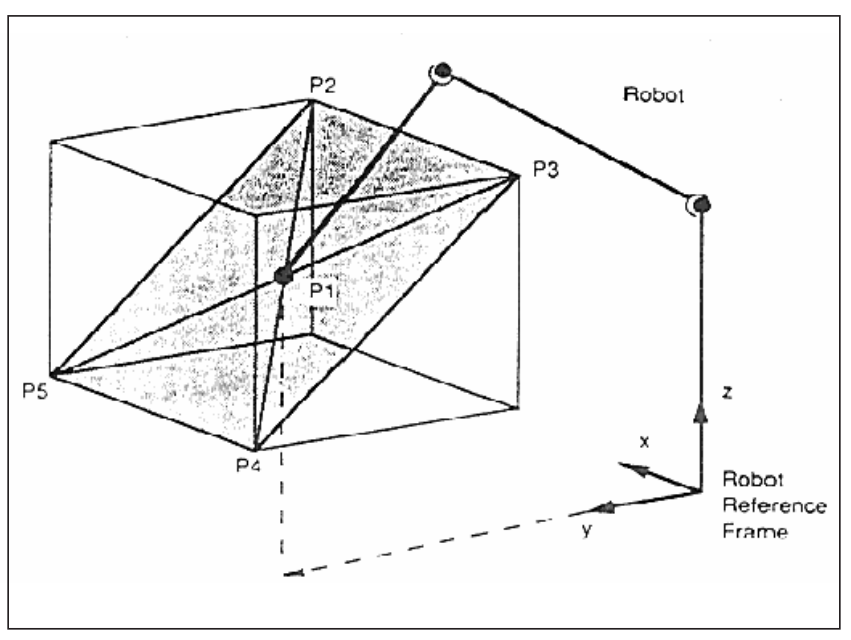

Figure 19

Spatial Path for Robot Calibration

\section{Robot Spatial Position Error Measurement}

According to the guideline for robot spatial path position error calibration (ISO 9283; 1990), the measuring sequence of a standard spatial paths $\mathrm{P} 1 \rightarrow \mathrm{P} 2 \rightarrow \mathrm{P} 3 \rightarrow \mathrm{P} 4 \rightarrow \mathrm{P} 5 \rightarrow \mathrm{P} 1$ is illustrated in Figure 19.

Experimental results are shown in Figure 20. The repeatability $(6 \sigma)$ of spatial position errors of the investigated robot is about $0.08 \sim 0.085 \mathrm{~mm}$, which meets general requirements. The maximum spatial position error is about $1 \mathrm{~mm}$, which implies that this robot is only suitable for rugged work.

\section{Conclusions}

A novel 3D-LBB system was developed for measuring the volumetric errors of multiaxis machines. This paper describes how possible errors due to as- 


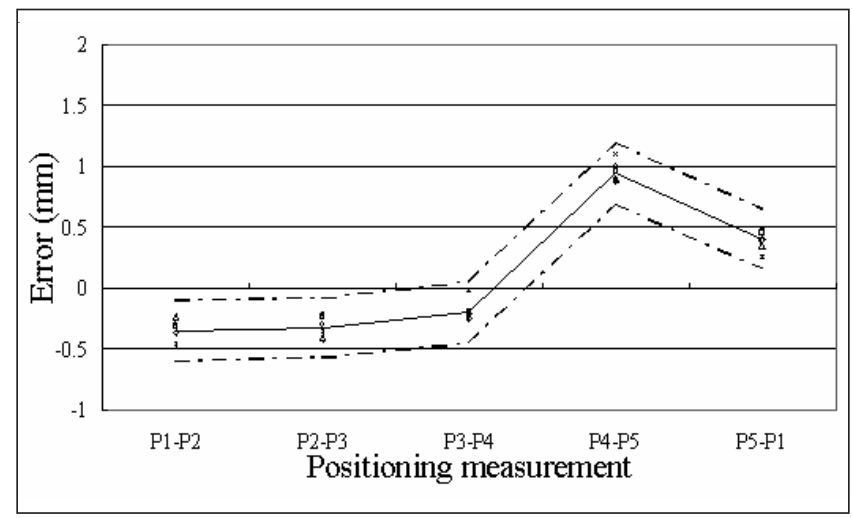

Figure 20

Results of a Robot Calibration

sembly, elastic deformation, and calibration processes could be found and compensated. For the current prototype, its accuracy after compensation is found to be quite promising: $\delta_{R}= \pm 0.3 \mu \mathrm{m}, \delta_{\phi}=$ \pm 1.5 arc-sec, and $\delta_{\theta}= \pm 1.6$ arc-sec. The repeatability and verification tests are also reported, which showed quite satisfactory results. This paper proposes the methodology of design, assembly, and error correction of a 3D laser ball bar, which has more flexible and versatile functions than the currently used laser interferometer and 1-D LBB for the accuracy calibration of machine tools. In addition to the spatial position measurements, this instrument also can be used to check other kinds of errors in machine tool metrology, such as circular test, straightness, spindle drift, and so on.

\section{References}

API Co. (2002). "Laser tracking system." http://www.apisensor.com. ASME B5.54 (1993). "Methods for performance evaluation of computer numerically controlled machining centers." Version 1.0.

Bryan, J.B. (1982). "A simple method for testing measuring machines and machine tools." Precision Engg. (v4, n2).

Burdekin, M. and Jywe, W. (1992). "Optimising the contouring accuracy of CNC machines using the Contisure system." Proc. of 29th Int'l MATADOR Conf.

ISO 230-4 (1998). "Acceptance code for machine tools, part 4circular measurements."

ISO 9283 (1990). "Manipulating industrial robots."
Parenti, V. and Gregorio, R.D. (1999). "Determination of the actual configuration of the general Stewart platform using one additional sensor." Journal of Mechanical Design, Trans. of ASME (v121, n1), pp21-25.

Patel, A.J. and Ehmann, K.F. (1997). "Volumetric error analysis of a modified Stewart platform-based machine tool." Annals of the CIRP (v46, n7), pp287-290.

Schmitz, T. and Ziegert, J. (2000). "Dynamic evaluation of spatial CNC contouring accuracy." Precision Engg. (v24), pp99-118.

Soons, J.A., Theuws, F.C., and Schellekens, P.H. (1992). "Modeling the errors of multi-axis machines: a general methodology." Precision Engg. (v14, n1), pp5-19.

Wang, C. (2000). "Laser vector measurement technique for the determination and compensation of volumetric positioning errors. Part 1: basic theory." American Institute of Physics, pp3933-3937.

Ziegert, J.C. and Mize, C.D. (1994). "The laser ball bar: a new instrument for machine tool metrology." Precision Engg. (v16, n4), pp259-267.

\section{Authors' Biographies}

Kuang-Chao Fan received the BSc degree from National Taiwan University (NTU) in 1972, MSc degree from the State University of New York at Buffalo in 1976, and PhD degree from the University of Manchester Institute of Science and Technology in 1984, all in mechanical engineering. He has been a professor of mechanical engineering at National Taiwan University since August 1989. He was the chairman of Institute of Industrial Engineering (from August 1994 to July 1995) and the associate dean of the engineering college at National Taiwan University (from August 1999 to July 2000). His research interests include manufacturing metrology, precision machining, and machine tool technology. He has published more than 100 technical papers. He was the recipient of the Outstanding Research Award 2003 by the National Science Council, Taiwan. He is the member of SME and ASPE.

Hai Wang received the BSc degree from National Taiwan University of Technology in 1986 and MSc degree and PhD degree from the Dept. of Mechanical Engineering at National Taiwan University in 1990 and 2004, respectively. He is an associate professor of mechanical engineering at Ming-Chi Institute of Technology. His research interests are precision machining and manufacturing metrology.

Fang-Jung Shiou received the MS degree from National Taiwan University and the $\mathrm{PhD}$ degree from the University of Technology at Aachen, Germany, both in mechanical engineering, in 1990 and 1998, respectively. He worked at the Laboratory for Machine Tools and Production Engineering (WZL) at the University of Technology at Aachen as a research assistant for four years. He is now with National Taiwan University of Science and Technology, Taipei, working as an associate professor in the Dept. of Mechanical Engineering. His major research interests include engineering metrology, precision machining, automatic optical inspection (AOI), and reverse engineering.

Chih-Wei Ke received his BS degree from Yuan-Ze University of Taiwan in 1996 and MSc degree from National Taiwan University in 2000 , both in mechanical engineering. He is an engineer at Richip Co, an IC design company. 